\title{
INFLUENCIA DEL NITRÓGENO EN LA CALIDAD DEL GRANO DE SORGO EN EL SALVADOR ${ }^{1}$
}

\author{
Vilma Ruth Calderón-Chinchilla ${ }^{2}$, Máximo Hernández-Valle ${ }^{3}$, Stephen Carl Mason ${ }^{4}$, Lloyd W. Rooney ${ }^{5}$
}

\begin{abstract}
RESUMEN
Influencia del nitrógeno en la calidad del grano de sorgo en El Salvador. Se evaluó el efecto de la aplicación y el uso eficiente de nitrógeno (UEN) en el rendimiento y la calidad del grano de 12 líneas experimentales de sorgo [Sorghum bicolor Moench (L)] y dos variedades comerciales ('Soberano' y 'Jocoro') durante el año 2004. Los niveles de nitrógeno empleados fueron de 0 y $21 \mathrm{~kg} / \mathrm{ha}$. Se presentaron interacciones entre las variedades y los niveles de nitrógeno para el rendimiento del grano y el UEN. La aplicación de 21 $\mathrm{kg} / \mathrm{ha}$ aumentó el rendimiento del grano en 0,9 t/ha (45\%), la concentración de $\mathrm{N}$ en el grano en $0,3 \%$ y la densidad verdadera del grano, con un efecto pequeño en otras variables de la calidad del grano. Se encontraron diferencias entre las líneas para todas las variables de calidad, pero ninguna de las líneas experimentales fue superior a las variedades Soberano y Jocoro. Las líneas ICSVLM-90520, ICSVLM-89537 e ICSVLM-90510 dieron rendimientos similares a Soberano y Jocoro; y ICSVLM-90510 presentó igual o superior calidad del grano, pudiendo ser utilizada para incrementar la diversidad genética.
\end{abstract}

Palabras claves: Uso eficiente de nitrógeno (UEN), densidad verdadera, densidad de volumen, prueba de dureza para granos individuales, instrumento abrasivo para descortezar el grano tangencialmente.

\begin{abstract}
Influence of nitrogen in the grain yield and quality of sorghum lines in El Salvador. The objective of this research was to determine the effect of the application of 0 and $21 \mathrm{~kg} / \mathrm{ha}$ of nitrogen fertilizer on grain yield, nitrogen use efficiency (NUE), and grain quality of 12 experimental grain sorghum [Sorghum bicolor Moench (L)] varieties and the commercial varieties Jocoro and Soberano during the growing season (fall 2004). Significant interactions between varieties and nitrogen application were only found for grain yield and NUE. Nitrogen application of $21 \mathrm{~kg} / \mathrm{ha}$ increased grain yield by $0,9 \mathrm{t} / \mathrm{ha}(45 \%)$, grain nitrogen concentration by $0,3 \%$ and true grain density, but had a small effects on other grain quality parameters. Differences were also observed among varieties in grain quality, but none of the experimental varieties was superior to Jocoro and Soberano, widely grown in El Salvador. The varieties ICSVLM-90520, ICSVLM89537 and ICSVLM-90510 had yields similar to Jocoro and Soberano, and ICSVLM-90510 had equal or superior grain quality, and could be used in a plant breeding programs to increase genetic diversity.
\end{abstract}

Key words: Nitrogen use efficiency (UEN), true density, bulk density (test weight), hardness (single kernel hardness test - SKHT), de-hulling device (Tangential Abrasive De-hulling Device -TADD).

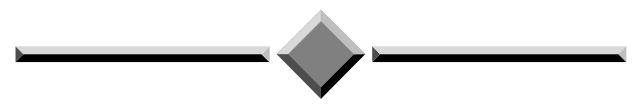

\footnotetext{
1 Recibido: 7 de junio, 2007. Aceptado: 18 de enero, 2008. Contribución del Centro Nacional de Tecnología Agropecuaria y Forestal, San Salvador, El Salvador; Departamento de Agronomía y Horticultura, Universidad de Nebraska, Lincoln, NE 68583-0915, EE.UU.; y Departamento de Cultivos y Suelos. Universidad de Texas A \& M, College Station, TX 77843-2474, EE.UU. Investigación financiada parcialmente por USAID (Donación No. DAN 1254-G-0021) a través del INTSORMIL (Programa Colaborativo Internacional de Sorgo y Mijo).

2 Laboratorio de Calidad de Cereales. Universidad de Texas A \& M. College Station TX, 77843-2474, EE.UU. Correo electrónico: vilmaruth@ yahoo.com

3 Investigador, Centro Nacional de Tecnología Agropecuaria y Forestal (CENTA) Km. 33 1/2 Carretera a Santa Ana, San Andrés, La Libertad, El Salvador. Correo electrónico: maxhernandezv@yahoo.com

4 Departamento de Agronomía y Horticultura. Universidad de Nebraska, P.O. Box 830915. Lincoln NE 68583-0915, EE.UU. Correo electrónico: smason1@unl.edu

5 Departmento de Cultivos y Suelos. Universidad de Texas A y M. College Station, TX, 77843-2474, EE.UU. Correo electrónico: 1rooney@ tamu.edu
} 


\section{INTRODUCCIÓN}

El sorgo de grano es el tercer cultivo básico más importante en El Salvador, el cual es sembrado generalmente por agricultores de bajos recursos económicos, en suelos poco fértiles y con poca o ninguna aplicación de fertilizantes. Incrementar el uso eficiente del nitrógeno (UEN) en el sorgo y su rendimiento de grano, en sorgo es importante para aumentar la rentabilidad del cultivo. Tradicionalmente, el grano de sorgo se ha utilizado como alimento para las aves de corral y los cerdos, pero en los últimos años el sorgo blanco ha contribuido a la elaboración de alimentos nixtamalizados como tortillas (Almeida-Dominguez et al. 1991), substituyendo la harina de trigo en productos de panadería (Rooney 2005), lo cual ha aumentado la necesidad de producir y poner a la disposición del mercado, grano de buena calidad.

Se han publicado muchos estudios relacionados con el UEN en el sorgo. Castañeda et al. (2006) reportaron diferencias varietales en el rendimiento del grano y el UEN con bajos niveles de fertilización en variedades sensibles al fotoperíodo. Los autores encontraron una alta respuesta al UEN en la variedad ICSVLM-90520, lo que generó la recomendación de sólo una aplicación de nitrógeno de 47 kg/ha. García et al. (2002) documentaron la respuesta en variedades insensibles al fotoperíodo y encontraron pequeñas diferencias, menores de las que han sido reportadas para variedades sensibles (Castañeda et al. 2006). Resultados similares fueron reportados en Africa (Bationo y Vlek 1998), Estados Unidos de América (Younquist et al. 1992) y Australia (Muchow y Sinclair 1994). El rendimiento del grano de sorgo se incrementó con la aplicación de fertilizante, pero la respuesta al nitrógeno varió con el nivel de aplicación (Pineda y Vanegas 2004), los cultivos previos (Peterson y Varvel 1989, Kaye et al. 2007a, 2007b), y la dosis de nitrógeno en el suelo (Bagayoko et al. 1992, Kaye et al. 2007b). La eficiencia del uso de nitrógeno, generalmente declina con el incremento de los niveles de nitrógeno (Buah et al. 1998).

La calidad del grano en el sorgo generalmente se ha enfocado a plantas con grano blanco y glumas de color canela. Se han reportado diferencias en el contenido nutricional (Singh y Axtell 1973, Oria et al. 2000) y en la calidad física (Rooney y Miller 1982, O’Brien
1999) en diferentes variedades. Estudios de fertilización han demostrado incrementos en la concentración de nitrógeno en el grano con el incremento en los niveles de nitrógeno aplicados (Hanson et al. 1988, Kamoshita et al. 1998). Johnson (2005) analizó muestras de grano de sorgo provenientes de Texas, Kansas y Nebraska encontró que el grano de sorgo es más duro y rinde menos cuando se produce bajo condiciones de alta temperatura y sequía lo cual había ocurrido en Texas; ésto se debe a la deposición del almidón que se ve restringida durante el llenado del grano cuando las temperaturas son elevadas, lo cual hace que se incremente la temperatura de gelatinización del almidón y la entalpía (Tester y Karkalas 2001). Kaye et al. (2007b) encontraron que las secuencias de los cultivos de sorgo en monocultivo y asocio con maíz y las enmiendas de fertilizantes, incrementan el contenido de $\mathrm{NO}_{3}-\mathrm{N}$ en el suelo, aumentando ligeramente el peso del grano y la densidad verdadera del grano de sorgo, pero al descortezar el grano, se disminuye significativamente el contenido de nitrogeno al expresarse en un gran aumento lo que queda del remanente TADD (afrecho de sorgo o corteza).

El principal objetivo de este estudio fue determinar la influencia de la aplicación de nitrógeno en la calidad del grano, el rendimiento y el UEN en 12 líneas experimentales de sorgo.

\section{MATERIALES Y MÉTODOS}

\section{Rendimiento y Uso Eficiente del Nitrogeno (UEN)}

Este estudio se realizó en las localidades de Suchitoto (Cuscatlán), Barrio Nuevo (San Vicente), San Miguel (San Miguel) y Tejutepeque (Cabañas) en El Salvador, durante el año 2004. Los suelos de estas regiones se caracterizan por tener bajo $\mathrm{pH}$, y bajo contenido de fósforo, y niveles de intermedio a altos de materia orgánica y potasio (Cuadro 1). El diseño experimental consistió en bloques completos al azar con arreglo en parcelas divididas y tres repeticiones. Las parcelas grandes estuvieron constituidas por 12 líneas experimentales del ICRISAT y las variedades comerciales Jocoro y Soberano de El Salvador (Cuadro 2). Como sub-parcela se consideraron las dosis de $\mathrm{N}$ de 0 y $21 \mathrm{~kg} / \mathrm{ha}$; incorporando $10,5 \mathrm{~kg} / \mathrm{ha}$ de sulfato de amonio, 30 días 
Cuadro 1. Características de los suelos y la precipitación, para cuatro localidades experimentales. El Salvador, 2004.

\begin{tabular}{lcccc}
\hline & San Miguel & Barrio Nuevo & Suchitoto & Tejutepeque \\
\hline Materia orgánica (\%) & 2,4 & 1,2 & 2,3 & 1,9 \\
$\mathrm{pH}$ & 5,6 & 4,9 & 5,5 & 4,7 \\
Fósforo (ppm) & 5 & 2 & 1 & 12 \\
Potasio (ppm) & 131 & 118 & 198 & 237 \\
Textura & Areno-Arcilloso & Areno-arcilloso & Areno-arcilloso & Areno- \\
& limoso & limoso & limoso & limoso \\
Precipitación $(\mathrm{mm})$ & 741 & 716 & 745 & 750 \\
\hline
\end{tabular}

Cuadro 2. Efecto del nitrógeno en el grano y rastrojo de 12 variedades de sorgo. El Salvador, 2004.

\begin{tabular}{|c|c|c|c|c|c|c|c|c|c|c|}
\hline \multirow[b]{3}{*}{ Línea } & \multicolumn{2}{|c|}{ Rendimiento } & \multicolumn{2}{|c|}{$\begin{array}{l}\text { Concentración de } \\
\text { nitrógeno }\end{array}$} & \multicolumn{2}{|c|}{$\begin{array}{l}\text { Absorción de } \\
\text { nitrógeno }\end{array}$} & \multicolumn{2}{|c|}{$\begin{array}{l}\text { Uso Eficiente del } \\
\text { Nitrógeno (UEN) }\end{array}$} & \multirow{3}{*}{$\begin{array}{c}\text { Altura } \\
\mathbf{c m}\end{array}$} & \multirow{3}{*}{$\begin{array}{c}\text { Floración } \\
\text { días }\end{array}$} \\
\hline & Grano & Rastrojo & Grano & Rastrojo & Grano & Rastrojo & Grano & Biomasa & & \\
\hline & \multicolumn{2}{|c|}{ t/ha } & \multicolumn{2}{|c|}{$\%$} & \multicolumn{2}{|c|}{ kg/ha } & \multicolumn{2}{|c|}{ kg/ha/kg/ha N } & & \\
\hline \multicolumn{11}{|l|}{ Experimental: } \\
\hline ICSVLM-93077 & 2,2 & 4,3 & 1,37 & 0,54 & 33 & 25 & 39 & 90 & 131 & 66 \\
\hline ICSVLM-90520 & 3,0 & 4,3 & 1,25 & 0,49 & 45 & 22 & 52 & 78 & 122 & 65 \\
\hline ICSVLM-89513 & 2,7 & 3,3 & 1,26 & 0,53 & 33 & 18 & 54 & 78 & 124 & 67 \\
\hline ICSVLM-92512 & 2,3 & 3,9 & 1,27 & 0,55 & 32 & 24 & 41 & 84 & 107 & 67 \\
\hline ICSVLM-93076 & 2,3 & 3,5 & 1,32 & 0,50 & 30 & 18 & 50 & 86 & 128 & 64 \\
\hline ICSVLM-89537 & 2,6 & 4,5 & 1,28 & 0,52 & 44 & 24 & 41 & 81 & 132 & 68 \\
\hline ICSVLM-93079 & 2,3 & 4,1 & 1,32 & 0,47 & 32 & 21 & 45 & 97 & 147 & 65 \\
\hline ICSVLM-89524 & 2,5 & 3,6 & 1,27 & 0,47 & 34 & 22 & 51 & 72 & 121 & 66 \\
\hline ICSVLM-92522 & 2,3 & 3,4 & 1,24 & 0,58 & 30 & 18 & 50 & 76 & 113 & 65 \\
\hline ICSVLM-90510 & 2,6 & 3,9 & 1,21 & 0,49 & 34 & 19 & 50 & 83 & 125 & 66 \\
\hline ICSVLM-93081 & 2,3 & 3,0 & 1,29 & 0,55 & 34 & 18 & 48 & 71 & 118 & 61 \\
\hline ICSVLM-93065 & 1,8 & 3,0 & 1,31 & 0,54 & 25 & 16 & 46 & 77 & 141 & 59 \\
\hline \multicolumn{11}{|l|}{ Testigos: } \\
\hline Soberano & 2,9 & 3,7 & 1,35 & 0,55 & 37 & 21 & 53 & 75 & 117 & 65 \\
\hline Jocoro & 2,5 & 4,2 & 1,20 & 0,50 & 34 & 28 & 44 & 84 & 136 & 67 \\
\hline DMS $(0,05)$ & 0,61 & 0,85 & NS & NS & 13,1 & 8,9 & 9,1 & 17,6 & 13,3 & 2,9 \\
\hline
\end{tabular}

DMS = indica diferencia mínima significativa

antes de la siembra y $10,5 \mathrm{~kg} / \mathrm{ha}$ en forma de urea, 60 días después de la siembra. El área de la parcela experimental fue de $16 \mathrm{~m}^{2}$, con cuatro surcos de $5 \mathrm{~m}$ de largo espaciados $0,80 \mathrm{~m}$. Las parcelas fueron raleadas a los 15 días para obtener una densidad de población de 109.890 plantas/ha. El control de malezas se hizo mediante una aplicación de $2 \mathrm{~kg} / \mathrm{ha}$ de Atrazina [(6-chloro-N-ethylN'-(1-methyl)-1,3,5-triazine-2, 4 diamine]) después de la siembra y también eliminándolas manualmente.

Las variables consideradas fueron: días a floración (d), altura de la planta $(\mathrm{cm})$ al momento de la madurez 
fisiológica, la unidad experimental fue número de panojas en $8 \mathrm{~m}^{2}$. Las sub-muestras de panojas y rastrojos se utilizaron para secarlas y calcular el rendimiento del grano y del rastrojo en base seca; posteriormente se analizó la concentración de nitrógeno. El peso seco del grano, del rastrojo, y el porcentaje de nitrógeno se utilizaron para determinar el uso eficiente del nitrógeno (UEN) (Maranville et al. 1980).

El UEN del grano y del rastrojo, junto con el rendimiento del grano y la biomasa se analizaron estadísticamente con un modelo de análisis mixto (Proc Mixed) (Littell et al. 1996), considerando como factores fijos las localidades, las variedades y los niveles de nitrógeno. Una diferencia mínima significativa con probabilidad de $\mathrm{P} \leq 0,05$ se utilizó con el fin de separar las medias por localidad y variedad.

\section{Calidad de grano}

Las muestras de grano de las doce líneas analizadas más los testigos, detallados en la sección de materiales y métodos (Cuadro 2), provenientes de cada repetición fueron analizadas para estudiar la calidad de grano para alimento. Los análisis de calidad se realizaron en el laboratorio de Calidad de Grano de la Universidad de Texas A y M, en College Station, Texas, EE.UU. El tamaño de muestra para los análisis de calidad fue de dos libras de grano por variedad.

Las muestras de grano se limpiaron para un análisis en triplicado, que se llevó a cabo para realizar el análisis proximal que expresa la concentración de almidón y de proteína, las propiedades físicas expresadas en la densidad verdadera y la aparente, el color del grano y su dureza. Las muestras se mantuvieron bajo refrigeración entre y durante los análisis.

La densidad verdadera del grano se obtuvo mediante el desplazamiento del gas nitrógeno utilizando un multi-picnómetro (Quantachrome Instruments, modelo MVP1 Syosset NY). La densidad verdadera es un índice que expresa la proporción de endospermo corneo y harinoso en el grano. Densidades con un rango de 1,2 a $1,4 \mathrm{~g} / \mathrm{cc}$ son normales en el grano de sorgo (Awika et al. 2002). La densidad aparente o densidad de volumen (test weight) se determinó en grano de sorgo limpio, de acuerdo a los estándares y procedimientos aprobados por el USDA-FGIS (2006). Valores de $620 \mathrm{~kg} \mathrm{~m}^{-3} \mathrm{o}$ más altos indican buena calidad del grano. El color del endospermo del grano se determinó con los valores $\boldsymbol{L}$, $\boldsymbol{a}$ y $\boldsymbol{b}$ dados por el colorimetro Chroma Meter CR300 (Konica Minolta, Tokyo, Japón). $\boldsymbol{L}$ es un índice que determina el brillo/claridad de la muestra, donde el color negro tiene un valor de 0 y el blanco de 100. El valor $\boldsymbol{a}$ mide los colores rojo $(\mathrm{a}+)$ y verde (a-) mientras que el $\boldsymbol{b}$ indica los colores amarillo (b+) y azul (b-) (Awika et al. 2002).

La dureza de las muestras en granos de sorgo limpio se determinó mediante la prueba de dureza para granos individuales (en inglés single kernel hardness tester - SKHT, Model SKCS 4100, Perten Instrument, Reno, NV). La fuerza requerida para triturar 300 granos, el diámetro y el peso, fueron reportados por Waniska y Rooney (2002). Un porcentaje de dureza mayor al 75\% indica que el grano es duro. El perlado o descortezado del grano se realizó con el método de la abrasión (en inglés tangential abrasive dehulling device -TADD, Model 4E-115, Creative Technologies, I.C. UTah). El porcentaje de grano descortezado es un índice de la calidad de la molienda (Rooney y Clark 1998), y un alto remanente TADD indica que los granos son duros, lo cual es deseado para la molienda seca (Shandera et al. 1997). El análisis proximal se realizó con la ayuda de la espectroscopia infrarroja cercana determinando así la concentración del almidón y la proteína.

Los factores de calidad del grano para las tres repeticiones se analizaron estadísticamente mediante el diseño de bloques completamente al azar con arreglo de parcelas divididas, considerando las localidades como repeticiones. Una diferencia mínima significativa con una probabilidad de $\mathrm{P} \leq 0,05$ se utilizó con el fin de separar las medias por variedad. Los coeficientes de correlación Pearson se calcularon para determinar las asociaciones entre rendimiento del grano y los diferentes parámetros de calidad obtenidos.

\section{RESULTADOS Y DISCUSIÓN}

\section{Localidad}

Se encontraron diferencias en los tipos de suelo (Cuadro 1) y en las variables agronómicas estudiadas (Cuadro 2), pero debido al alto grado de variación entre 
las localidades (Cuadro 3), la separación de los efectos por localidad fue difícil de interpretar estadísticamente. En todas las localidades, los suelos presentaron bajo el $\mathrm{pH}$ y bajo contenido de fósforo. Los más bajos $\mathrm{pH}$ se dieron en Barrio Nuevo y Tejutepeque, y en Barrio Nuevo los suelos con más bajos contenido de materia orgánica (Cuadro 1). El rendimiento de la biomasa fue mayor en San Miguel y Tejutepeque mientras que el rendimiento de rastrojo fue más alto en San Miguel (Cuadro 3). El mejor UEN para biomasa se encontró en Barrio Nuevo con suelos menos fértiles (Cuadro 1), mientras que el más bajo UEN para grano se obtuvo en San Miguel (Cuadro 3).

\section{Dosis de Nitrógeno}

La aplicación de $21 \mathrm{~kg} /$ ha de nitrógeno, incrementó el rendimiento del grano y del rastrojo, la concentración del nitrógeno en el grano, la absorción del nitrógeno y la altura de la planta, mientras que aceleró la madurez y redujo el UEN de la biomasa (Cuadro 4). Los resultados fueron consistentes con los obtenidos por Buah et al. (1998), Hanson et al. (1988), y Kamoshita et al. (1998), pero la respuesta fue mucho más alta para el rendimiento del grano (45\%) y del rastrojo (34\%) con la aplicación de nitrógeno que para la concentración, absorción y el UEN.

\section{Variedad}

Se encontraron diferencias varietales para todos las variables medidas excepto para la concentración de nitrógeno en el grano y el rastrojo (Cuadro 2). Hubo interacción variedad por dosis de nitrógeno en el rendimiento del grano y el UEN en el grano (datos no presentados). Ninguna de las líneas experimentales fue superior a los testigos, los cuales son comercialmente producidos en El Salvador (Deras y Castañeda 2002).

Cuadro 3. Concentración, absorción y uso eficiente del nitrógeno, y el rendimiento de grano y del rastrojo de sorgo en cuatro localidades. El Salvador, 2004.

\begin{tabular}{|c|c|c|c|c|c|c|c|c|c|c|}
\hline \multirow[b]{3}{*}{ Localidad } & \multicolumn{2}{|c|}{ Rendimiento } & \multicolumn{2}{|c|}{$\begin{array}{c}\text { Concentración de } \\
\text { nitrógeno }\end{array}$} & \multicolumn{2}{|c|}{$\begin{array}{l}\text { Absorción de } \\
\text { nitrógeno }\end{array}$} & \multicolumn{2}{|c|}{$\begin{array}{l}\text { Uso eficiciente del } \\
\text { nitrógeno(UEN) }\end{array}$} & \multirow{3}{*}{$\begin{array}{c}\text { Altura } \\
\text { cm }\end{array}$} & \multirow{3}{*}{$\begin{array}{c}\text { Floración } \\
\text { días }\end{array}$} \\
\hline & Grano & Rastrojo & Grano & Rastrojo & Grano & Rastrojo & Grano & Biomasa & & \\
\hline & \multicolumn{2}{|c|}{ t/ha } & \multicolumn{2}{|r|}{$\%$} & \multicolumn{2}{|c|}{ kg/ha } & \multicolumn{2}{|c|}{ kg/ha/kg/ha N } & & \\
\hline San Miguel & 3,3 & 5,7 & 1,40 & 0,65 & 46 & 37 & 40 & 72 & 137 & 58 \\
\hline Barrio Nuevo & 1,2 & 2,6 & 1,10 & 0,36 & 18 & 11 & 49 & 114 & 133 & 76 \\
\hline Suchitoto & 2,3 & 3,5 & 1,19 & 0,45 & 29 & 16 & 53 & 84 & 118 & 63 \\
\hline Tejutepeque & 3,1 & 3,3 & 1,43 & 0,62 & 43 & 20 & 48 & 53 & 116 & 65 \\
\hline D.M.S. $(0,05)$ & 1,50 & 1,53 & 0,092 & 0,116 & 17,8 & 13,1 & 10,8 & 44,4 & 13,5 & 7,3 \\
\hline
\end{tabular}

DMS = indica diferencia mínima significativa.

Cuadro 4. Influencia del nitrógeno en el grano y rastrojo de sorgo, en cuatro localidades. El Salvador, 2004.

\begin{tabular}{|c|c|c|c|c|c|c|c|c|c|c|}
\hline \multirow{3}{*}{$\begin{array}{c}\text { Nivel de } \\
\text { nitrógeno } \\
\text { kg/ha }\end{array}$} & \multicolumn{2}{|c|}{ Rendimiento } & \multicolumn{2}{|c|}{$\begin{array}{c}\text { Concentración de } \\
\text { nitrógeno }\end{array}$} & \multicolumn{2}{|c|}{$\begin{array}{c}\text { Absorción de } \\
\text { nitrógeno }\end{array}$} & \multicolumn{2}{|c|}{$\begin{array}{l}\text { Uso eficiente del } \\
\text { nitrógeno (UEN) }\end{array}$} & \multirow{3}{*}{$\begin{array}{c}\text { Altura } \\
\text { cm }\end{array}$} & \multirow{3}{*}{$\begin{array}{c}\text { Floraciór } \\
\text { días }\end{array}$} \\
\hline & Grano & Rastrojo & Grano & Rastrojo & Grano & Rastrojo & Grano & Biomasa & & \\
\hline & t/ha & & $\%$ & kg/ha & & & \multicolumn{2}{|c|}{$\mathrm{kg} \mathrm{ha}^{-1 / \mathrm{kg} \mathrm{ha}^{-1} \mathrm{~N}}$} & & \\
\hline 0 & 2,0 & 3,2 & 1,22 & 0,48 & 27 & 18 & 47 & 87 & 122 & 68 \\
\hline 21 & 2,9 & 4,3 & 1,25 & 0,50 & 42 & 25 & 48 & 75 & 130 & 62 \\
\hline \multicolumn{11}{|l|}{ ANDEVA } \\
\hline Significancia & $<0,01$ & $<0,01$ & 0,02 & NS & $<0,01$ & $<0,01$ & NS & $<0,01$ & $<0,01$ & $<0,01$ \\
\hline
\end{tabular}


Las líneas ICSVLM-90520, ICSVLM-89537 e ICSVLM-90510 tuvieron rendimientos similares a los testigos, por lo que se pueden incorporar a los programas de fitomejoramiento para incrementar la diversidad genética.

\section{Calidad de grano}

\section{Nitrógeno}

En general, el efecto de la aplicación de nitrógeno aumentó bastante el rendimiento (Cuadro 4) pero influyó poco en la calidad del grano, y no se encontró interacción entre la variedad y la aplicación de nitrógeno, lo cual puede apreciarse en el Cuadro 6, donde se correlacionan las variables de calidad de grano con los rendimientos. Este resultado difiere del obtenido por Awika et al. (2002) quienes reportaron que las aplicaciones de nitrógeno influyeron significativamente en incrementos en el rendimiento y en la densidad verdadera del grano. El incremento en la densidad verdadera se debe al aumento de la concentración de la proteína en el grano lo cual ocurre con las aplicaciones de nitrógeno (Hanson et al. 1988, Kamoshita et al. 1998). Sin embargo, los resultados son consistentes con estudios llevados a cabo en trigo (Triticum aestivum L.), donde se encontró que pequeñas cantidades del fertilizante nitrogenado, en situaciones donde el nitrógeno es limitante, aumentan significativamente el rendimiento mientras que mantienen un efecto muy pequeño en la concentración de la proteína (Bezian y Lane 1979). Por otra parte, Kaye et al. (2007a) reportaron que en el sorgo, las secuencias de cultivos y las enmiendas en el suelo que incrementan el $\mathrm{NO}_{3}-\mathrm{N}$ aumentan ligeramente el peso del grano y la densidad verdadera del grano, y también aumentan el remanente TADD substancialmente.

\section{Variedad}

Se encontraron diferencias entre las líneas en todas las variables relacionados con la calidad del grano excepto con la densidad verdadera (Cuadro 5). Ninguna de las líneas experimentales mostró un color de grano más blanco y brillante que el de las variedades comerciales, pero ICSVLM-90520, ICSVLM-89524 e ICSVLM-90510 presentaron brillantez estadísticamente igual al testigo Jocoro. Granos blancos brillantes y plantas con glumas de color canela, generan productos alimenticios atractivos y deseados por el consumidor (Waniska y Rooney 2002).

Ninguna de las líneas examinadas presentó concentraciones de almidón más altas que la de los testigos, aunque se encontró una gran variación en la concentración de la proteína (Cuadro 5). Las líneas ICSVLM93065 e ICSVLM-92512 tuvieron las concentraciones de proteína más altas, pero bajos rendimientos (Cuadro 2); contrario a las líneas ICSVLM-90520, ICSVLM89524 y Soberano, las cuales mostraron las concentraciones de proteínas más bajas (Cuadro 5) pero los rendimientos más altos (Cuadro 2). Estos resultados son consistentes con anteriores investigaciones en cereales, donde se reportó una relación inversa entre el rendimiento del grano y la concentración de la proteína (Mason y D'Croz-Mason 2002).

No se encontraron diferencias en la densidad verdadera entre las líneas; la densidad de volumen y las diferencias del remanente TADD fueron pequeñas comportándose los testigos mejor que las líneas experimentales (Cuadro 5). En la prueba SKHT, Jocoro presentó, estadísticamente, el grano más duro, pero ICSVLM90510 e ICSVLM-93076 no fueron significativamente menos duras. Soberano exhibió el peso SKHT más grande y un diámetro más pequeño que Jocoro. Las líneas ICSVLM-92522, ICSVLM-93077 e ICSVLM93079 fueron más pesadas que los testigos y que otras líneas, mientras que ICSVLM-93065 e ICSVLM-93081 presentaron el diámetro más grande, sin ser significativamente más grande que ICSVLM-93079

Las características ideales que debe tener una variedad de sorgo con calidad de grano para alimento son alto rendimiento, glumas de color canela, y granos blancos, brillantes y duros (Rooney 1996). Los resultados de este estudio indican que las variedades comerciales Soberano y Jocoro poseen la mayoría de estas características (Cuadro 2 y 5). Las líneas experimentales ICSVLM-90520 e ICSVLM-89513 dieron un rendimiento similar y/o superior a los testigos pero con calidad del grano baja. En general ISCVLM-90510 mostró una calidad de grano igual o superior a los testigos Soberano y Jocoro y con un rendimiento similar (Cuadro 2), indicando que posee potencial para desarrollar líneas en un programa de fitomejoramiento. Las otras líneas ocasionalmente mostraron superioridad 
Cuadro 5. Diferencia en la calidad del grano en las líneas de sorgo en cuatro localidades, en El Salvador, 2004.

\begin{tabular}{|c|c|c|c|c|c|c|c|c|c|c|c|}
\hline \multirow[b]{3}{*}{ Línea } & \multicolumn{3}{|c|}{ Color } & \multicolumn{2}{|c|}{ Densidad } & \multirow[b]{2}{*}{ Proteína } & \multirow[b]{2}{*}{ Almidón } & \multirow{3}{*}{$\begin{array}{c}\text { TADD } \\
\% \\
\end{array}$} & \multicolumn{3}{|c|}{ SKHT } \\
\hline & $\mathbf{a}$ & $\mathbf{b}$ & $\mathbf{L}$ & Aparente & Verdadera & & & & \multirow{2}{*}{$\begin{array}{c}\text { Dureza } \\
\text { escala }\end{array}$} & \multirow{2}{*}{$\begin{array}{c}\text { Peso } \\
\mathrm{mg}\end{array}$} & \multirow{2}{*}{$\begin{array}{c}\text { Diámetro } \\
\mathrm{mm} \\
\end{array}$} \\
\hline & & & & $\mathrm{kg} \mathrm{m}^{-3}$ & $\mathrm{~g} \mathrm{cc}^{-3}$ & & 6 & & & & \\
\hline ICSVLM-93077 & 4,5 & 16,4 & 57,9 & 709 & 1,395 & 8,9 & 78,4 & 80,4 & 69,9 & 28,8 & 2,39 \\
\hline ICSVLM-90520 & 3,0 & 16,2 & 62,1 & 713 & 1,395 & 7,4 & 80,3 & 82,1 & 80,3 & 25,7 & 2,01 \\
\hline ICSVLM-89513 & 4,2 & 18,2 & 60,1 & 713 & 1,382 & 8,2 & 78,7 & 81,2 & 72,2 & 27,8 & 2,22 \\
\hline ICSVLM-92512 & 10,7 & 14,2 & 48,0 & 716 & 1,402 & 9,8 & 76,8 & 84,4 & 80,6 & 27,7 & 2,17 \\
\hline ICSVLM-93076 & 3,9 & 17,7 & 59,5 & 718 & 1,398 & 7,8 & 79,6 & 84,8 & 89,8 & 23,8 & 1,96 \\
\hline ICSVLM-89537 & 4,0 & 16,7 & 59,9 & 716 & 1,388 & 7,8 & 78,7 & 83,1 & 80,7 & 24,4 & 1,72 \\
\hline ICSVLM-93079 & 4,1 & 16,0 & 58,8 & 714 & 1,400 & 8,5 & 79,2 & 83,0 & 78,9 & 29,0 & 2,28 \\
\hline ICSVLM-89524 & 3,2 & 17,3 & 62,7 & 708 & 1,398 & 7,4 & 80,5 & 83,2 & 82,8 & 24,4 & 1,92 \\
\hline ICSVLM-92522 & 10,2 & 15,2 & 48,6 & 703 & 1,389 & 8,4 & 77,6 & 78,3 & 75,6 & 29,0 & 2,05 \\
\hline ICSVLM-90510 & 3,6 & 17,2 & 61,9 & 730 & 1,405 & 7,8 & 80,1 & 83,1 & 88,6 & 24,1 & 1,83 \\
\hline ICSVLM-93081 & 4,2 & 16,5 & 58,7 & 705 & 1,391 & 9,1 & 78,5 & 77,2 & 74,2 & 27,2 & 2,30 \\
\hline ICSVLM-93065 & 4,2 & 16,2 & 58,5 & 699 & 1,389 & 9,7 & 78,4 & 77,3 & 75,2 & 27,8 & 2,41 \\
\hline SOBERANO & 2,9 & 16,7 & 63,4 & 719 & 1,406 & 7,4 & 80,2 & 81,4 & 80,7 & 26,7 & 2,09 \\
\hline JOCORO & 3,2 & 17,7 & 62,1 & 726 & 1,413 & 8,8 & 79,3 & 85,4 & 93,4 & 23,4 & 1,78 \\
\hline DMS $(0,05)$ & 0,41 & 0,91 & 1,53 & 15,6 & NS & 0,84 & 0,79 & 4,55 & 5,25 & 1,69 & 0,119 \\
\hline
\end{tabular}

DMS = indica diferencia mínima significativa

en la calidad del grano para uno u otra variable, pero carecieron de consistencia para otras variables, lo cual sugiere que estas variedades de grano no son adecuadas para usos alimenticios.

Relaciones entre las líneas de la calidad del grano

Aunque muchos de los factores de calidad dieron coeficientes de correlación significativos, sólo algunas de estas correlaciones fueron lo suficientemente altas para ser de importancia (Cuadro 6). Se observó una correlación alta pero negativa entre la concentración de la proteina y la del almidón, lo cual fue reportado previamente (Hoseney 1994, Waniska y Rooney 2002). Hoseney (1994) encontró que el almidón es más denso que la proteína y que bajo condiciones normales se sedimenta más extensamente que la proteina. Una correlación alta y positiva se observó entre el peso SKHT y el diámetro SKHT. Así un grano grande debe pesar más si las diferencias en la densidad verdadera son pequeñas, como se observó en este estudio (Cuadro 5).
Correlaciones intermedias $(>0,40$ a $<0,60)$ se observaron en la dureza SKHT y la densidad aparente SKHT y el remanente TADD (Cuadro 6) lo cual tiene sentido porque los tres son variables que miden la dureza del grano. Se encontró una correlación negativa entre la dureza SKHT y el diámetro SKHT confirmando de esta manera que los granos pequeños son más duros lo cual fue previamente reportado en maíz (Johnson y Russell 1982). La asociación entre el rendimiento del grano y los parámetros de calidad fue por lo general baja, sugiriendo que los programas de fitomejoramiento en el sorgo deben tratar de desarrollar variedades que posean estas dos cualidades, buen potencial de rendimiento y una alta calidad de grano.

\section{CONCLUSIONES}

Diferencias varietales se encontraron en las variables estudiadas excepto para la concentración de nitrógeno en el rastrojo. Tan solo se encontraron interacciones entre las líneas y las dosis de nitrógeno para el 
Cuadro 6. Coeficientes de correlación entre el rendimiento y las variables de calidad de grano promediado para 12 líneas de sorgo y cuatro localidades. El Salvador, 2004.

\begin{tabular}{|c|c|c|c|c|c|c|c|c|}
\hline \multirow[t]{2}{*}{ Parámetros } & \multirow[t]{2}{*}{ Proteína } & \multirow[t]{2}{*}{ Almidón } & \multicolumn{2}{|c|}{ Densidad } & \multirow{2}{*}{$\begin{array}{c}\text { TADD } \\
\text { removal }\end{array}$} & \multirow{2}{*}{$\begin{array}{c}\text { SKHT } \\
\text { Dureza } \\
\end{array}$} & \multirow{2}{*}{$\begin{array}{c}\text { SKHT } \\
\text { Peso } \\
\end{array}$} & \multirow{2}{*}{$\begin{array}{c}\text { SKHT } \\
\text { Diámetro }\end{array}$} \\
\hline & & & Aparente & Verdadera & & & & \\
\hline Rendimiento & $-0,36^{* *}$ & 0,12 & 0,18 & $0,24 *$ & $0,20 *$ & $0,32 * *$ & $0,29 * *$ & 0,16 \\
\hline Proteína & & $-0,91 * *$ & $-0,14$ & 0,05 & 0,11 & 0,04 & $0,32 * *$ & $0,35 * *$ \\
\hline Almidón & & & 0,04 & 0,11 & $-0,09$ & 0,09 & 0,09 & $-0,13$ \\
\hline Densidad aparente & & & & 0,14 & $0,32 * *$ & $0,47 * *$ & $-0,37$ & $-0,33$ \\
\hline Densidad verdadera & & & & & $0,22 *$ & 0,15 & 0,10 & 0,04 \\
\hline TADD removal & & & & & & $0,45^{* *}$ & $-0,26^{* *}$ & $-0,28 * *$ \\
\hline SKHT Dureza & & & & & & & $-0,39 * *$ & $-0,47 * *$ \\
\hline SKHT Peso & & & & & & & & $0,84 * *$ \\
\hline SKHT Diámetro & & & & & & & & $-0,02$ \\
\hline
\end{tabular}

$* \mathrm{y} * *$ indican significancia al $\mathrm{P} \leq 0,05$ y $\mathrm{P} \leq 0,01$.

rendimiento del grano y el UEN en el grano. Ninguna de las variedades fue superior a los testigos, Soberano y Jocoro comercialmente producidos en El Salvador. Las líneas ICSVLM-90520, ICSVLM-89537 y ICSVLM90510 tuvieron rendimientos similares a los testigos y podrían ser incorporadas en los programas de fitomejoramiento para incrementar la diversidad genética.

La aplicación de $21 \mathrm{~kg} / \mathrm{ha}$ de $\mathrm{N}$ incrementó un $45 \%$ el rendimiento del grano (0,9 t/ha) y un $34 \%$ el del rastrojo $(1,1 \mathrm{t} / \mathrm{ha})$ mientras que el efecto sobre las variables de calidad fue mínimo. En la mayoría de los aspectos de producción, esta gran respuesta al aumento del rendimiento con este bajo nivel de aplicación de $\mathrm{N}$ podría traducirse en beneficios para el productor. $\mathrm{La}$ selección de variedades con alto rendimiento y alta calidad del grano en combinación con aplicaciones de nitrógeno contribuirán a satisfacer la demanda por alimentos para el consumo humano en El Salvador.

\section{LITERATURA CITADA}

Almeida-Dominguez, HD; Serna-Salvidar, SO; Rooney, LW. 1991. Properties of new and commercial sorghum hybrids for use in alkaline-cooked foods. Cereal Chemistry 68(1): 25-30.

Awika, J; Suhendro, L; Elly, L; Rooney, L. 2002. Milling values of sorghums compared by adjusting yields to a constant product color. Cereal Chemistry 79(2): 249-251.

Bagayoko, M; Mason, SC; Sabata, RJ. 1992. Residual effects of cropping systems on soil nitrogen and grain sorghum yields. Agronomy Journal 83: 862-835.

Bationo, A; Vlek, P. 1998. The role of nitrogen fertilizers applied to food crops in the Sudano-Sahelian zone of West Africa, p. 43-51. In: Renard, G.; Neef, A.; Becker, K; von Open, M. eds. Soil fertility management in West African land use systems. Margraf Verlag, Weikersheim, Germany. 599 p.

Bezian, B; Lane, P. 1979. Some relationships between grain yield and grain production of wheat experiments in south-east England and comparisons with such relationships elsewhere. Journal of the Science of Food and Agriculture 30: 59 - 70 .

Buah, S; Maranville, J; Traore, A; Brammel-Cox, P. 1998. Response of nitrogen use efficient sorghums to nitrogen fertilizer. Journal of Plant Nutrition 21: 23032318.

Castañeda, JW; Mason, SC; Argueta, Q; Ventura, R; Hernández Valle, M; Clará, R. 2006. Eficiencia del uso de nitrógeno en sorgo sensible al fotoperiodo en asocio con maíz en El Salvador. Revista Agronomía Mesoamericana 17(2): 19-25. 
Deras, H; Castañeda, W. 2002. Guía técnica del sorgo en monocultivo para grano. CENTA, San Andrés, El Salvador.

Duodu, KG; Taylor, JRN; Belton, PS; Hamaker, BR. 1990. Factors affecting sorghum protein digestibility. Cereal Chemistry 32: 117-131.

García, L; Téllez, O; Mason, S. 2002. Determinación del uso eficiente de nitrógeno en cuatro variedades de sorgo para grano en la zona del Pacifico de Nicaragua. Revista La Calera 3: 36-42.

Hahn, R. 1999. Dry milling of sorghum grain. Journal of Cereal Science 14: 234-237.

Hanson, RG; Stecker, JA; Maledy, SR. 1988. Effect of soybean rotation on the response of sorghum fertilizer nitrogen. Journal of Production Agriculture 1: 318 $-321$.

Hoseney, C. 1994. Principles of cereal science and technology. 2 ed. American Association of Cereal Chemistry. St. Paul, MN. 389 p.

Johnson, W. 2005. Corn and sorghum characteristics influencing wet milling and nixtmalization performance. M.S. Thesis, University of Nebraska, Lincoln, NE. 85 p.

Johnson, DG; Russell, WA. 1982. Genetic variability and relationships of physical grain quality traits in the BSSS population of maize. Crop Science 22: 805-809.

Kamoshita, S; Fukai, S; Muchow, RC; Cooper, M. 1998. Genotypic variation for grain yield and grain nitrogen concentration among sorghum hybrids under different levels of nitrogen fertilizer and water supply. Australian Journal of Agricultural Research 49: 737747.

Kaye, NM; Mason, SC; Jackson, DJ; Galusha, TD. 2007a. Crop rotation and soil amendment alters sorghum grain quality. Crop Science 47: 722-729.

Kaye, NM; Mason, SC; Galusha, TD; Mamo, M. 2007b. Nodulating and non-nodulating soybean rotation influence on soil $\mathrm{NO}_{3}-\mathrm{N}$ and water, and sorghum grain yield. Agronomy Journal 99: 599-606.
Littell, RC; Milliken, GA; Stroup, WW; Wolfinger, RD. 1996. SAS system for mixed models. SAS Institute, Cary, North Carolina. 633 p.

Maranville, JW; Clark, RB; Ross, W. 1980. Nitrogen use efficiency in grain sorghum. Journal of Plant Nutrition 2:577-589.

Mason, SC; D'Croz-Mason, NE. 2002. Agronomic practices influence maize grain quality. Journal of Crop Production 5: 75-91.

Muchow, RC; Sinclair, TR. 1994. Nitrogen response of leaf photosynthesis canopy radiation use efficiency in field-grown maize and sorghum. Field Crops Research 18: 17-30.

O'Brien, L. 1999. Genotype and environment effects on feed grain quality. Austrailian Journal of Agricultural Research 50: 703-720.

Oria, MP; Hamaker, BK; Axtell, JD; Huang, C. 2000. A highly digestible sorghum cultivar exhibits a unique folded structure of endosperm protein bodies. Proceeding of the National Academy of Science 97(10): 5065-5070.

Pineda, L; Vanegas, A. 2004. Cultivo de sorgo. Guía Tecnológica 5, Instituto Nicaragüense de Tecnología Agropecuaria (INTA), Managua, Nicaragua. 15 p.

Peterson, TA; Varvel, GE. 1989. Crop yield as affected by crop rotation and $\mathrm{N}$ rate. II. Sorghum. Agronomy Journal 81: 727-731.

Rooney, W; Miller, DS. 1982. Sorghum grain quality. In: Proceedings of the International Symposium on Sorghum Grain Quality. ICRISAT Center, Patancheru, India. $125 \mathrm{p}$.

Rooney, LW. 1996. Attributes of improved quality sorghums for value-added marketing. American Seed Trade Association. Proceedings of the $51^{\text {st }}$ Annual Corn and Sorghum Research Conference. Chicago, IL. p. 112-124.

Rooney, LW. 2005. Food and nutritional quality of sorghum and millet. In INTSORMIL 2005 Annual Report. Lincoln, NE 68583-0748. p. 103-112.

Rooney, LW; Clark, L. 1998. Chemistry and processing of sorghum grain. Journal of Cereal Science 13(7): 258-265. 
Shandera, DL; Jackson, DS; Johnson, BE. 1997. Quality factors impacting processing of maize dent hybrids. Maydica 42: 282-289.

Singh, R; Axtell, JD. 1973. High lysine mutant gene (hl) that improves protein and biological value of grain sorghum. Crop Science 13: 535-539.

USDA-FGIS. 2006. Test weight per bushel apparatuses. Chapter 5. Equipment Handbook. United States Department of Agriculture - Federal Grain Inspection Service, Washington, DC. 28-34 p.
Waniska, R; Rooney, LW. 2002. Sorghum grain quality for increased utilization. Sorghum and Millet Diseases. Leslie, J.F. ed. Iowa State Press, Ames, IA. p. 327-335.

Tester, R; Karkalas, F. 2001. The effects of environmental conditions on structural and physico-chemical properties of starch. Starch/Stärke 53: 513-519.

Youngquist, J; Brammel-Cox, P; Maranville, JW. 1992. Evaluation of alternate screening criteria for selecting nitrogen use efficient genotypes in sorghum. Crop Science 32: 1310-1313. 